九州大学学術情報リポジトリ

Kyushu University Institutional Repository

\title{
Molecular Cloning of the Genes Involved in Anthocyanin Biosynthesis in Camellia japonica
}

Tateishi, Nobumine

Laboratory of Horticultural Science, Division of Agricultural Botany, Department of Plant Resources, Graduate School of Bioresource and Bioenvironmental Sciences, Kyushu University

Ozaki, Yukio

Laboratory of Agricultural Ecology, Division of Agricultural Ecology, Department of Plant Resources, Faculty of Agriculture, Kyushu University

Okubo, Hiroshi

Laboratory of Agricultural Ecology, Division of Agricultural Ecology, Department of Plant Resources, Faculty of Agriculture, Kyushu University

https://doi.org/10.5109/17796

出版情報: 九州大学大学院農学研究院紀要. 55 (1)，pp.21-28，2010-02-26. Faculty of Agriculture， Kyushu University

バージョン :

権利関係 : 


\title{
Molecular Cloning of the Genes Involved in Anthocyanin Biosynthesis in Camellia japonica
}

\section{Nobumine TATEISHI ${ }^{1 *}$, Yukio OZAKI ${ }^{2}$ and Hiroshi OKUBO}

Laboratory of Horticultural Science, Division of Agricultural Botany, Department of Plant Resources, Faculty of Agriculture, Kyushu University, Fukuoka 812-8581, Japan

(Received October 26, 2009 and accepted November 19, 2009)

\begin{abstract}
Partial cDNA sequences of three anthocyanin biosynthetic genes (F3H, flavanone 3-hydroxylase; DFR, dihydroflavonol 4-reductase; ANS, anthocyanidin synthase) were isolated from the petals of Camellia japonica. Their deduced partial amino acid sequences shared high homologies with those of woody plant species (CjF3Ha, 98.0\%, CjF3Hb, 91.2\% and CjDFR, 99.0\% with Camellia sinensis; CjANS, $90.3 \%$ with Rhododendron $\times$ pulchrum). Some important amino acid residues for enzymatic activities were also conserved in the isolated clones, suggesting that the genes we identified in this study were the homologues of C. japonica. Gene-specific primer pairs were designed based on each partial cDNA sequence. The application of these primer pairs to RT-PCR analyses was tested.
\end{abstract}

\section{INTRODUCTION}

The primary reason of the bright flower colours of plants is to be a signal to attract insects and birds for successful pollination by emphasising their floral tissue against a background of vegetation (Glover, 2007). The major pigments that cause flower colour are carotenoids, flavonoids and betalains (Davies, 2009). Flavonoids also have some additional important functions, like defence against pathogens and predators, prevention from damaging by UV light, action as antioxidants, and involvement in pollen development and germination (Glover, 2007). Anthocyanins are one of the plant-derived flavonoid compounds and are responsible for colours ranging from pink and red to purple and deep blue (Deroles, 2009). Most detailed studies about anthocyanin biosynthesis have been achieved in Antirrhinum and Petunia as models and many genes involved in the biosynthetic pathway have been isolated so far (Martin et al., 1991; Holton and Cornish, 1995).

Camellia japonica has a long history as a representative woody ornamental plant in Japan (Tuyama, 1968a). Wild type C. japonica has a single-petalled red flowers, and two major anthocyanins were identified in the petals, namely cyanidin 3-glucoside and cyanidin 3-galactoside (Sakata et al., 1986, 1987). However, molecular mechanism dominating their flower pigmentation has not been reported, and the breeding program of camellia plants still depends on the selection of the seedlings that appear by chance. Molecular information controlling flower characters of camellia must contribute to an efficient breeding system of the plant.

Laboratory of Horticultural Science, Division of Agricultural Botany, Department of Plant Resources, Graduate School of Bioresource and Bioenvironmental Sciences, Kyushu University, Fukuoka 812-8581, Japan

${ }^{2}$ Laboratory of Agricultural Ecology, Division of Agricultural Ecology, Department of Plant Resources, Faculty of Agriculture, Kyushu University, Fukuoka 811-2307, Japan

* Corresponding author (E-mail: nobumine2006@yahoo.co.jp)
In this paper, we isolated partial cDNA sequences of three anthocyanin biosynthetic genes in C. japonica, flavanone 3-hydroxylase (F3H), dihydroflavonol 4-reductase (DFR) and anthocyanidin synthase (ANS), and confirmed that their gene-specific primer pairs are available for RT-PCR analyses.

\section{MATERIALS AND METHODS}

\section{Plant materials}

Fully expanded flowers of wild $C$. japonica accession (collected in Kurose, Fukue Island, Nagasaki Prefecture, Japan), 'Tamanoura' and 'Hatsu-arashi' were picked immediately at the beginning of anthesis. The petals of 'Tamanoura' were separated into red and white marginal picotee parts, and only the red parts were used in this study. The petals were frozen promptly using liquid nitrogen and stored at $-80^{\circ} \mathrm{C}$ until use for RNA extraction.

\section{RNA extraction}

RNA extraction was carried out following Kiefer et al. (2000)'s manner with some modifications. Frozen tissues (100-150 mg) were ground to fine powder with mortar and pestle using liquid nitrogen and $800 \mu \mathrm{l}$ prewarmed $\left(65^{\circ} \mathrm{C}\right)$ extraction buffer $[100 \mathrm{mM}$ Tris-HCl $(\mathrm{pH}$ 8.0), $25 \mathrm{mM}$ EDTA, $2 \mathrm{M} \mathrm{NaCl}, 2 \% \mathrm{CTAB}$ (w/v), $2 \%$ polyvinylpyrrolidone (w/v), $0.5 \%$ spermidine (w/v) and $2 \%$ ß-mercaptoethanol (v/v)] were added followed by incubation at $65{ }^{\circ} \mathrm{C}$ for $10 \mathrm{~min}$. One hundred $\mu \mathrm{l}$ of Nucleon PhytoPure DNA extraction resin (GE Healthcare, England) and $400 \mu \mathrm{l}$ of chloroform/isoamylalcohol (24:1) were added and the sample tubes were kept on a shaker for $10 \mathrm{~min}$ at room temperature. After centrifugation at $9,000 \times$ g for 10 min at $4{ }^{\circ} \mathrm{C}$, aqueous phase was washed with $500 \mu \mathrm{l}$ of chloroform/isoamylalcohol (24:1) at least three times. The aqueous phase, to which $500 \mu \mathrm{l}$ of isopropanol were added, were incubated on ice for $1 \mathrm{~h}$, followed by centrifugation at $9,000 \times \mathrm{g}$ for $10 \mathrm{~min}$ at $4{ }^{\circ} \mathrm{C}$. RNA pellets were dissolved in $100 \mu \mathrm{l}$ DEPC water with 
$2 \mathrm{M}$ of $\mathrm{LiCl}$ and incubated at $4{ }^{\circ} \mathrm{C}$ overnight. After centrifugation at $9,000 \times \mathrm{g}$ for $30 \mathrm{~min}$ at $4{ }^{\circ} \mathrm{C}$, the RNA pellets were treated with DNase I (Roche Diagnostics, Germany) at $37^{\circ} \mathrm{C}$ for $20 \mathrm{~min}$. Equal volume of isopropanol were added for RNA precipitation, followed by centrifugation at $9,000 \times$ g for 2 min at $4{ }^{\circ} \mathrm{C}$. After two times wash with $70 \% \mathrm{EtOH}$, the pellets were dried and dissolved in DEPC water. The RNA concentration and purity were evaluated with the absorbance at $260 \mathrm{~nm}$

\section{RT-PCR analyses}

cDNA synthesis and RT-PCR were carried out using TaKaRa RNA PCR Kit (AMV) Ver. 3.0 (Takara, Japan) following the manufacturer's instructions. We mixed two kinds of primers in the ratio of Oligo dT-adaptor primer:Random 9 mers $=4: 1$ and used for reverse tran-

Table 1. Primer pairs designed and used in this study

\begin{tabular}{|c|c|c|c|}
\hline Primer & Gene & & Sequence \\
\hline \multirow[t]{6}{*}{ Degenerate primer } & F3H & Forward & 5'-ATG TCC GGT GGB AAR AAR GG-3' \\
\hline & & Reverse & 5'-TTG CTC ATC TTC YTC YKG TAC-3' \\
\hline & DFR & Forward & 5'-RAG GAY CCY GAG AAT GAR G-3' \\
\hline & & Reverse & 5'-GCT GTA YTT GAA YTY GAA YCC-3' \\
\hline & $A N S$ & Forward & 5'-TTG AGT GGS AGG ATT AYT TYT TYC-3' \\
\hline & & Reverse & 5'-GGT TCG CAG AAV ATH GCC C-3' \\
\hline \multirow[t]{6}{*}{ Gene-specific primer } & СjF3Ha & Forward & 5'-ACG GAG ACC TAC AGC GAG AA-3' \\
\hline & & Reverse & 5’-ATG ATC CGC ATT CTT GAA CC-3' \\
\hline & CjDFR & Forward & 5'-AAC AAC CCA TTT TCG ACG AG-3' \\
\hline & & Reverse & 5'-TTG TAC TCG GGC CAT TTC TC-3' \\
\hline & CjANS & Forward & 5'-ACG CAA AGC AAC TAC GAG G-3' \\
\hline & & Reverse & 5'-CTA CCG TGT CAC CAA TGT GC-3' \\
\hline
\end{tabular}

$\mathrm{B}=\mathrm{C}+\mathrm{G}+\mathrm{T} ; \mathrm{R}=\mathrm{A}+\mathrm{G} ; \mathrm{Y}=\mathrm{C}+\mathrm{T} ; \mathrm{K}=\mathrm{G}+\mathrm{T} ; \mathrm{S}=\mathrm{C}+\mathrm{G} ; \mathrm{V}=\mathrm{A}+\mathrm{C}+\mathrm{G} ; \mathrm{H}=\mathrm{A}+\mathrm{C}+\mathrm{T}$

\begin{tabular}{|c|c|c|c|c|c|}
\hline 10 & 20 & 30 & 40 & 50 & 60 \\
\hline TGGATTCATC & GTTTCCAGTC & АTСTCCAGGG & AGAAGCAGTG & CAAGACTGGA & GAGAAATAGT \\
\hline 70 & 80 & 90 & 100 & 110 & 120 \\
\hline GАССТАСTTC & TCATACCCGA & TCCGGGCCCG & GGACTATTCA & AGATGGCCCG & ACAAGCCCGA \\
\hline 130 & 140 & 150 & 160 & 170 & 180 \\
\hline AGGGTGGAGG & GCTGTGACGG & $\begin{array}{r}\text { AGACCTACAG } \\
\text { СјF3Ha }\end{array}$ & $\begin{array}{l}\text { CGAGAAATTG } \\
\mathrm{F}\end{array}$ & ATGGACTTGG & СTTGCAAGTT \\
\hline 190 & 200 & 210 & 220 & 230 & 240 \\
\hline GCTGGAGGTG & TTGTCTGAGG & ССАTGGACCT & TGAGAAGGAG & GСТСТTАСАА & AAGCCTGTGT \\
\hline 250 & 260 & 270 & 280 & 290 & 300 \\
\hline TGATATGGAT & CAGAAGGTGG & TTGTAAATTT & СТАCCCGAAA & TGCCCACAAC & СCGACCTCAC \\
\hline 310 & 320 & 330 & 340 & 350 & 360 \\
\hline GCTCGGACTC & AAGCGACACA & CGGATCCGGG & ТTССАТСАСС & СтGСTCCTCC & AGGACCAGGT \\
\hline 370 & 380 & 390 & 400 & 410 & 420 \\
\hline TGGTGGGCTC & CAGGCCACTA & GAGATGGGGG & CAAGACCTGG & ATCACGGTTC & AGCCCGTGGA \\
\hline 430 & 440 & 450 & 460 & 470 & 480 \\
\hline GGGAGCTTTT & GTTGTTAATC & TGGGTGACCA & TGGTCATTAT & СТAAGCAATG & GGAGGTTCAA \\
\hline 490 & 500 & 510 & 520 & 530 & 540 \\
\hline GAATGCGGAT & CATCAGGCAG & TAGTGAACTC & CAACTGCAGC & СGACTATCAA & TCGCTACATT \\
\hline CjF3Ha ${ }_{550}^{\mathrm{R}}$ & 560 & 570 & 580 & 590 & 600 \\
\hline CCAGAACCCA & GCTCCCGAGG & CGACAGTATA & СССАСТGAAG & ATTAGGGAGG & GAGAGAAGCC \\
\hline 610 & 620 & 630 & 640 & & \\
\hline GTTC & & & & & \\
\hline
\end{tabular}

Fig. 1. Nucleotide sequence of partial cDNA of CjF3Ha. The shadowed boxes represent the primer regions of CjF3Ha $\mathrm{F}$ and $\mathrm{R}$ used in this study. 
scriptional reaction. PCR amplification was performed in a total volume of $50 \mu \mathrm{l}$ containing $45 \mathrm{ng}$ template cDNA, $0.2 \mu \mathrm{M}$ of each primer, $10 \mu \mathrm{l}$ of $5 \times$ PCR Buffer, $0.2 \mathrm{mM}$ of each dNTP, 1.25 Unit TaKaRa Ex Taq HS polymerase. In case of using degenerate primer pairs, their volumes were calculated according to its degeneracy (Table 1). Finally, RNase Free $\mathrm{H}_{2} \mathrm{O}$ was added up to $50 \mu \mathrm{l}$. Amplification was conducted using TaKaRa PCR Thermal Cycler Dice TP-600 (TaKaRa, Japan) with one cycle of $3 \mathrm{~min}$ at $94^{\circ} \mathrm{C}$, followed by 30 cycles of $20 \mathrm{sec}$ at $94^{\circ} \mathrm{C}$, $20 \mathrm{sec}$ at $56^{\circ} \mathrm{C}$ and $1 \mathrm{~min}$ at $72^{\circ} \mathrm{C}$, finally one cycle of $10 \mathrm{~min}$ at $72^{\circ} \mathrm{C}$. PCR products were separated by electrophoresis in 1.5\% (w/v) agarose gels and visualised under UV illumination after staining with ethidium bromide.

\section{Molecular cloning of $F 3 H, D F R$ and $A N S$ in $C$. japonica}

Degenerate RT-PCR products from wild C. japonica were subcloned into pGEM-T Easy Vector (Promega, USA) and transformed into Competent high Escherichia coli DH5 $\alpha$ (Toyobo, Japan). After culture on the LB plates containing $100 \mu \mathrm{g} / \mathrm{ml}$ of ampicillin, $100 \mu \mathrm{g} / \mathrm{ml}$ of $\mathrm{X}-\mathrm{Gal}$ and $23.83 \mu \mathrm{g} / \mathrm{ml}$ of IPTG, only white colonies were selected, and then the plasmids containing inserts were extracted using LaboPass Plasmid Mini Purification Kit (Hokkaido System Science, Japan). BigDye Terminator v1.1 Cycle Sequencing Kit (Applied Biosystems, USA) and ABI PRISM 310 genetic analyzer (Applied Biosystems, USA) were employed for sequence analyses. The deduced amino acid sequence alignments and phylogenetic trees were obtained by using DNASIS-Mac v3.2 (Hitachi Software Engineering, Japan) and PHYLIP version 3.6 (Felsenstein, 2004), respectively.

\section{RESULTS}

\section{Molecular cloning of $F 3 H, D F R$ and $A N S$ in $C$. japonica}

We identified $633 \mathrm{bp}$ fragment of partial CjF3H. Sequence analysis revealed that it contained multi-gene family. The major one was named CjF3Ha (GenBank accession no. AB524883) and minor one was $\mathrm{CjF} 3 \mathrm{Hb}$

CjF3Ha
CjF3Hb
Vitis
Gentiana
Ipomoea nil
Arabidopsis
Oryza sativa
CjF3Ha
CjF3Hb
Vitis
Gentiana
Ipomoea nil
Arabidopsis
Oryza sativa

CjF3Ha
CjF3Hb
Vitis
Gentiana
Ipomoea nil
Arabidopsis
Oryza sativa

\begin{tabular}{|c|c|c|c|c|}
\hline 10 & 20 & 30 & 40 & 50 \\
\hline GFIVSSHLQG & EAVQDWREIV & TYFSYPIRAR & DYSRWPDKPE & GWRAVTETYS \\
\hline GFIVSSHLQG & EAVQDWREIV & TYFSYPIRAR & DYSRWPDKPE & EWRAVTEKYS \\
\hline GFIVSSHLQG & EAVQDWREIV & TYFSYPLRTR & DYSRWPDKPE & GWRSVTQEYS \\
\hline GFIVSSHLQG & EAVRDWREIV & TYFSYPIKSR & DYSRWPDKPE & GWKSVTEKYS \\
\hline GFIVSSHLQG & EAVKDWREIV & TYFSYPVRAR & DYSRWPDKPE & GWRAVTEKYS \\
\hline GFIVSSHLQG & EAVQDWREIV & TYFSYPVRNR & DYSRWPDKPE & GWVKVTEEYS \\
\hline GFIVSSHLQG & EAVKDWREIV & TYFSYPVKSR & DYSRWPDKPA & GWRAVVEQYS \\
\hline 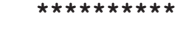 & & 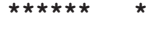 & 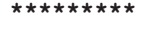 & $\star \star$ \\
\hline 60 & 70 & 80 & 90 & 100 \\
\hline
\end{tabular}

51 EKLMDLACKL LEVLSEAMDL EKEALTKACV DMDQKVVVNF YPKCPOPDL'

51 SSLMELACKL LEVLSEAMGL EKEALTNACV DMDQKVVVNY YPKCPQPDLT

51 EKLMGLACKL LEVLSEAMDL DKDALTNACV DMDOKVVVNF YPQCPOPDLT

51 EQLMNLACKL LEVLSESMRL EKEALTKACV DMDQKIVVNF YPKCPQPDLT

51 EKLMDLACKL LEVLSEAMGL EKEALSKACV ELDQKLVVNF YPKCPEPDLT

51 ERLMSLACKL LEVLSEAMGL EKESLTNACV DMDQKIVVNY YPKCPQPDLT

51 ERLMGLACKL LGVLSEAMGL DTNALADACV DMDQKVVVNF YPKCPQPDLT

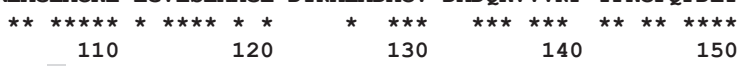

101 LGLKRHTDPG SITLLLQDQV GGLQATRDGG KTWITVQPVE GAFVVNLGDH

101 LGLKRHTDPG TITLLLODOV GGLOATRDGG KTWITVOPVE GAFVVNLGDH

101 LGLKRHTDPG TITLLLQDQV GGLQATRDGG KTWITVQPVE GAFVVNLGDH

101 LGLKRHTDPG TITLLLQDQV GGLQATRDGG KSWITVQPVD GAFVVNLGDH

101 LGLKRHTDPG TITLLLQDQV GGLQATKDGG KTWITVQPVD GAFVVNLGDH

101 LGLKRHTDPG TITLLLQDQV GGLQATRDNG KTWITVQPVE GAFVVNLGDH

101 LGLKRHTDPG TITLLLQDLV GGLQATRDAG KTWITVQPIP GSFVVNLGDH

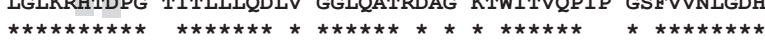

160

170

180

190

200

151 GH----- ---YLSNGRF KNADHQAVVN SNCSRLSIAT FQNPAPEATV 200

151 GH------ ---YLSNGRF KNADHQAVVN SNSSRLSIAT FQNPAPEAIV 200

151 GH------- --YLSNGRF KNADHQAVVN SNHSRLSIAT FQNPAPEATV 200

151 GH------- --YLSNGRF KNADHQAVVN SNYSRLSIAT FQNPAPEATV 200

151 GH------ ---FLSNGRF KNADHQAVVN SEHSRMSIAT FQNPAPEAKV 200

151 GH------- ---FLSNGRF KNADHQAVVN SNSSRLSIAT FQNPAPDATV 200

151 AHIMHLLGNV NLQYLSNGRF KNADHQAVVN SDCCRLSIAT FQNPAPDAMV 200

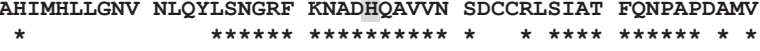
210

$220 \quad 230 \quad 240 \quad 250$

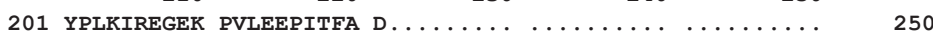

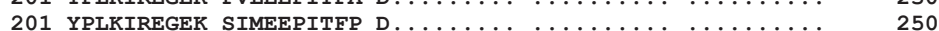

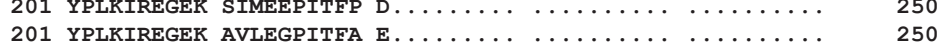

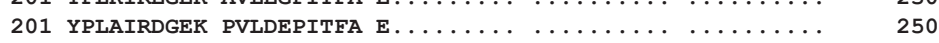

201 YPLKVREGEK PILEEPITFA E................... 250

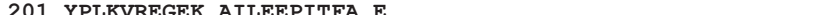

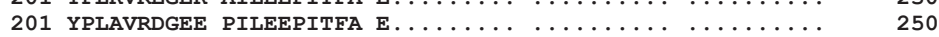

$$
\star * \star * \star * \quad * * \star *
$$

Fig. 2. Comparison of the deduced amino acid sequences of partial CjF3Hs and those of corresponding parts of other plant species. Asterisks indicate completely identical amino acid residues among six species. The shadowed amino acid residues (His106, Asp108 and His175) represent absolute conservation important for catalytic activity. Species names and their GenBank accession numbers are as follows; Arabidopsis (Arabidopsis thaliana, AF064064); Gentiana (Gentiana triflora, AB193311); Ipomoea nil (Ipomoea nil, D83041); Oryza sativa (Oryza sativa, NM001060692); Vitis (Vitis vinifera, EF192467). 


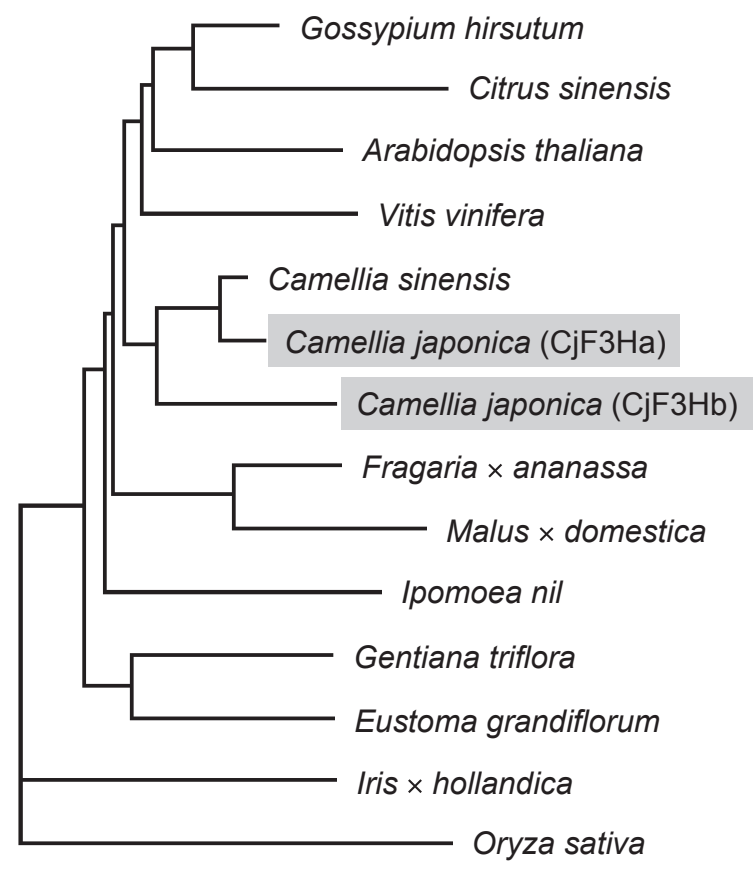

Fig. 3. A phylogenetic tree for F3H constructed by neighbor-joining methods using partial deduced amino acid sequences, including $\mathrm{CjF} 3 \mathrm{Ha}$ (AB524883), $\mathrm{CjF} 3 \mathrm{Hb}$ (AB524884) identified in this study. Sequence data were collected from GenBank databases [Arabidopsis thaliana (AF064064), Camellia sinensis (AY641730), Citrus sinensis (AB011795), Eustoma grandiflorum (AB078956), Fragaria $\times$ ananassa (AB201760), Gentiana triflora (AB193311), Gossypium hirsutum (EF187440), Ipomoea nil (D83041), Iris $\times$ hollandica (AB183826), Malus $\times$ domestica (AB074486), Oryza sativa (NM001060692), Vitis vinifera (EF192467)].

0.02

10

20

30

40

50

60

TAATCAAGCC GACAATCAAC GGTGTGTTGA GCATCATAAG GTCATGCACC AAAGCTAAGA

$\begin{array}{lllllr}70 & 80 & 90 & 100 & 110 & 120\end{array}$

CAGTGAAGAG GCTGGTGTTC ACATCCTCTG CTGGAACTGT TAATGTCCAG GAACACCAAC

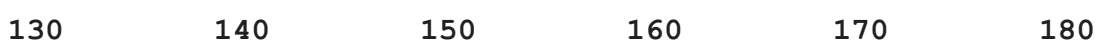

AACCCATTTT CGACGAGAAC AATTGGAGTG ACTTGGATTT CATCAATAAG AAGAAGATGA

CjDFR F

$\begin{array}{llrrr}190 & 200 & 210 & 220 & 230\end{array}$

CTGGCTGGAT GTATTTTGTT TCAAAAACAT TGgCAGAGAA AGCAGCATGg GAAGCAGCAA

$\begin{array}{llllll}250 & 260 & 270 & 280 & 290 & 300\end{array}$

AAGAGAACAA CATTGATTTC ATTAGTATCA TTCCTACATT AGTTGTAGGA CCTTTCATCA

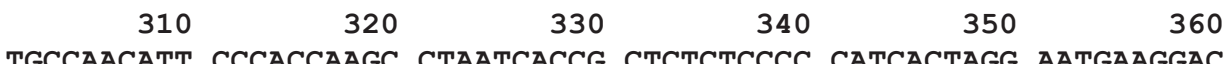

$\begin{array}{lllll}370 & 380 & 390 & 400 & 410\end{array}$

ACTATTCGAT CATAAAGCAA GGGCAGTTTG TGCACCTTGA TGATCTCTGT GAATCTCATA

$\begin{array}{llllll}430 & 440 & 450 & 460 & 470 & 480\end{array}$

TATTCTTGTA TGAGCATCCT CAGGCTGAGG GCAGATACAT TTGCTCCTCC CATGATGCTA

$\begin{array}{llrrr}490 & 500 & 510 & 520 & 530\end{array}$

CCATCCATGA TTTGGCCAAA CTGATGAGAG AGAAATGGCC CGAGTACAAT GTCCCCACTG

$\begin{array}{lllll}550 & 560 & 570 & \text { CjFR R } & 590\end{array}$

AGTTTAAGGG GATAGACAAG GACTTGCCAG TTGTGTCGTT CTCATCGAAG AAGTTGATAG

610

GAATG....

Fig. 4. Nucleotide sequence of partial cDNA of CjDFR. The shadowed boxes represent the primer regions of $C j D F R \mathrm{~F}$ and $\mathrm{R}$ used in this study. 


\begin{tabular}{|c|c|c|c|c|c|c|c|}
\hline & & 10 & 20 & 30 & 40 & 50 & \\
\hline CjDFR & 1 & IKPTINGVLS & IIRSCTKAKT & VKRLVFTSSA & GTVNVQEHQQ & PIFDENNWSD & 50 \\
\hline Vitis & 1 & IKPTIEGMLG & IMKSCAAAKT & VRRLVFTSSA & GTVNIQEHQL & PVYDESCWSD & 50 \\
\hline Malus & 1 & IKPTINGLLD & ILKACQKAKT & VRKLVFTSSA & GTVNVEEHQK & PVYDESNWSD & 50 \\
\hline Rosa & 1 & IKPTINGVLD & IMQACLKAKT & VRRLVFTSSA & GSVNVEETQK & PVYNESNWSD & 50 \\
\hline Gentiana & 1 & IKPTIDGFLS & I IRSCVKAKT & VKKLVFTSSA & GTVDVQEQQK & PVYDENDWSD & 50 \\
\hline Ipomoea nil & 1 & IKPAINGVLN & IINSCVKAKT & VKRLVFTSSA & GTLNVQPQQK & PVYDETCWSD & 50 \\
\hline Arabidopsis & 1 & IKPTVNGMLG & IMKACVKAKT & VRRFVFTSSA & GTVNVEEHQK & NVYDENDWSD & 50 \\
\hline \multirow[t]{3}{*}{ Oryza sativa } & 1 & VKPTVEGMLS & IMRACRDAGT & VKRIVFTSSA & GTVNIEERQR & PSYDHDDWSD & 50 \\
\hline & & 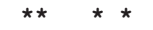 & 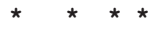 & 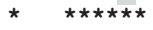 & * $\quad$ * & $\star \star \star \star$ & \\
\hline & & 60 & 70 & 80 & 90 & 100 & \\
\hline CjDFR & 51 & LDFINKKKMT & GWMYFVSKTL & AEKAAWEAAK & ENNIDFISII & PTLVVGPFIM & 100 \\
\hline Vitis & 51 & MEFCRAKKMT & AWMYFVSKTL & AEQAAWKYAK & ENNIDFITII & PTLVVGPFIM & 100 \\
\hline Malus & 51 & VEFCRSVKMT & GWMYFVSKTL & AEQAAWKYAK & ENNIDFITII & PTLVIGPFLM & 100 \\
\hline Rosa & 51 & VEFCRRVKMT & GWMYFASKTL & AEQEAWKFAK & ENNIDFITII & PTLVIGPFLM & 100 \\
\hline Gentiana & 51 & LDFINSTKMT & GWMYFVSKIL & AEKAAWEVTK & ANDIGFISII & PTLVVGPFIT & 100 \\
\hline Ipomoea nil & 51 & LDFIYAKKMT & GWMYFASKIL & AEKEAWKVTK & EKKIDFISII & PPLVVGPFIT & 100 \\
\hline Arabidopsis & 51 & LEFIMSKKMT & GWMYFVSKSL & AEKAAWDFAE & EKGLDFISII & PTLVVGPFIT & 100 \\
\hline \multirow[t]{3}{*}{ Oryza sativa } & 51 & IDFCRRVKMT & GWMYFVSKSL & AEKAAMEYAR & EHGLDLISVI & PTLVVGPFIS & 100 \\
\hline & & $\star \quad \quad * * *$ & $\star \star \star \star \star \star \star * \star *$ & $\star \star * \quad *$ & $\star \star$ & $\star \star \star \star \star \star * \star$ & \\
\hline & & 110 & 120 & 130 & 140 & 150 & \\
\hline CjDFR & 101 & PTFPPSLITA & LSPITRNEGH & YSIIKQGQFV & HLDDLCESHI & FLYEHPQAEG & 150 \\
\hline Vitis & 101 & SSMPPSLITA & LSPITGNEAH & YSIIRQGQFV & HLDDLCNAHI & YLFENPKAEG & 150 \\
\hline Malus & 101 & PSMPPSLITG & LSPILRNESH & YGIIKQ̈GQYYV & HLDDLCLSHI & YLYEHPKAEG & 150 \\
\hline Rosa & 101 & PSMPPSLITG & LSPLTGNESH & YSIIKQGQFI & HLDDLCQSHI & YLYEHPKAEG & 150 \\
\hline Gentiana & 101 & TTFPPSLITA & LSLITGNEAH & YGI IKQGQFV & HLDDLCEAHI & FLYEHPEAEG & 150 \\
\hline Ipomoea nil & 101 & PTFPPSLITA & LSLITGNQAH & YSIIKQGQYV & HLDDLCEAHI & FLYEHPKAEG & 150 \\
\hline Arabidopsis & 101 & TSMPPSLITA & LSPITRNEAH & YSIIRQGQYV & HLDDLCNAHI & FLYEQAAAKG & 150 \\
\hline \multirow[t]{3}{*}{ Oryza sativa } & 101 & NGMPPSHVTA & LALLTGNEAH & YSILKQVQFV & HLDDLCDAEI & FLFESPEARG & 150 \\
\hline & & $\star \star \star \quad \star \quad$ & $\star \quad \star \star *$ & 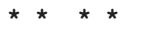 & $\star \star \star \star \star \star \star \quad \star \quad$ & 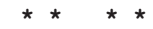 & \\
\hline & & 160 & 170 & 180 & 190 & 200 & \\
\hline CjDFR & 151 & RYICSSHDAT & IHDLAKLMRE & KWPEYNVPTE & FKGID-KDLP & VVSFSSKKLI & 200 \\
\hline Vitis & 151 & RYICSSHDCI & ILDLAKMLRE & KYPEYNIPTE & FKGVD-ENLK & SVCFSSKKLT & 200 \\
\hline Malus & 151 & RYICSSHDAT & IHELVKMLRE & KYPEYNIPTK & FKGID-DNLE & PVHFSSKKLR & 200 \\
\hline Rosa & 151 & RYICSSHDAT & IHEIAKLLKG & KYPEYNVPTT & FKGIE-ENLP & KVHFSSKKLL & 200 \\
\hline Gentiana & 151 & RYICSSHDTT & IHDLAKMIRQ & NWPEYYIPTK & LKGID-EDIP & VVSFSSNKLI & 200 \\
\hline Ipomoea nil & 151 & RFICSSHHTT & IHGLADMITQ & NWPEYYIPSE & FKGIE-KDLP & VVYFSSKKLQ & 200 \\
\hline Arabidopsis & 151 & RYICSSHDAT & ILTISKFLRP & KYPEYNVPST & FEGVD-ENLK & SIEFSSKKLT & 200 \\
\hline \multirow[t]{3}{*}{ Oryza sativa } & 151 & RYVCSSHDAT & IHGLATMLAD & MFPEYDVPRS & FPGIDADHLQ & PVHFSSWKLL & 200 \\
\hline & & $\star \quad \star \star \star \star \star *$ & $\star$ & $\star \star \star \quad \star \quad$ & * & 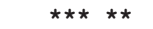 & \\
\hline & & 210 & 220 & 230 & 240 & 250 & \\
\hline CjDFR & 201 & GM. . . . . & $\ldots \ldots \ldots \ldots$ & $\ldots \ldots \ldots$ & $\ldots \ldots \ldots$ & $\ldots \ldots \ldots$ & 250 \\
\hline Vitis & 201 & DL $\ldots \ldots \ldots$ & $\ldots \ldots$ & & & & 250 \\
\hline Malus & 201 & EI. . & $\ldots \ldots$ & & & $\ldots$ & 250 \\
\hline Rosa & 201 & ЕT.... & $\ldots \ldots$ & $\cdots$ & $\cdots$ & $\ldots$ & 250 \\
\hline Gentiana & 201 & DL . . . & $\ldots \ldots$ & & . & $\ldots \ldots$ & 250 \\
\hline Ipomoea nil & 201 & DM....... & $\cdots \cdots$ & 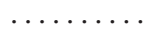 & & $\ldots \ldots \ldots$ & 250 \\
\hline Arabidopsis & 201 & EM. . . . & & & & $\ldots \ldots \ldots$ & 250 \\
\hline Oryza sativa & 201 & $\mathrm{AH}, \ldots \ldots$ & & & & $\ldots$ & 250 \\
\hline
\end{tabular}

Fig. 5. Comparison of the deduced amino acid sequence of partial CjDFR and those of corresponding parts of other plant species. Asterisks indicate completely identical amino acid residues among eight species. The shadowed amino acid residues (Ser29 and Tyr64) represent absolute conservation important for catalytic activity. Species names and their GenBank accession numbers are as follows; Arabidopsis (Arabidopsis thaliana, AB033294); Gentiana (Gentiana triflora, D85185); Ipomoea nil (Ipomoea nil, AB006792); Malus (Malus $\times$ domestica, AY227728); Oryza sativa (Oryza sativa, AB003496); Rosa (Rosa hybrida, D85102); Vitis (Vitis vinifera, X75964).

(GenBank accession no. AB524884), both encoded 210 amino acid residues (Figs. 1 and 2). Phylogenetic tree was constructed to compare the homology of deduced amino acid sequences of $\mathrm{CjF} 3 \mathrm{Ha}$ and $\mathrm{CjF} 3 \mathrm{Hb}$ with other related F3Hs (Fig. 3). CjF3Ha and $\mathrm{CjF} 3 \mathrm{Hb}$ shared 98.0\% and $91.2 \%$ identities with $\mathrm{F} 3 \mathrm{H}$ from Camellia sinensis, respectively.

The length of partial DFR clone of C. japonica was $605 \mathrm{bp}$, in which 201 amino acid residues were encoded (Figs. 4 and 5) (GenBank accession no. AB524885). Figure 6 shows the comparison of deduced amino acid sequences of partial DFRs. The deduced amino acid sequence of CjDFR showed high identity with that of Camellia sinensis (99.0\%).

Partial CjANS length we obtained was $468 \mathrm{bp}$ and it encoded 155 amino acid residues (Figs. 7 and 8)
(GenBank accession no. AB524886). The deduced amino acid sequence of CjANS clustered with those of woody plants, for instance, Rhododendron $\times$ pulchrum (90.3\%) (Fig. 9).

\section{Gene-specific RT-PCR}

We designed gene-specific primer pairs for each gene (CjF3Ha, CjDFR and CjANS) based on the cDNA sequences we obtained in this study (Figs. 1, 4, 7 and Table 1). Although two sequences in $\mathrm{F} 3 \mathrm{H}, \mathrm{CjF} 3 \mathrm{Ha}$ and $\mathrm{CjF} 3 \mathrm{Hb}$, were identified, $\mathrm{CjF} 3 \mathrm{Ha}$ was applied for the design of $C j F 3 H$ gene-specific primer, because of its frequent appearance in cloning procedure. Bands of expected sizes were amplified successfully in all samples, wild type C. japonica and 'Tamanoura' (red parts of the petals) and 'Hatsu-arashi' with RT-PCR using these 


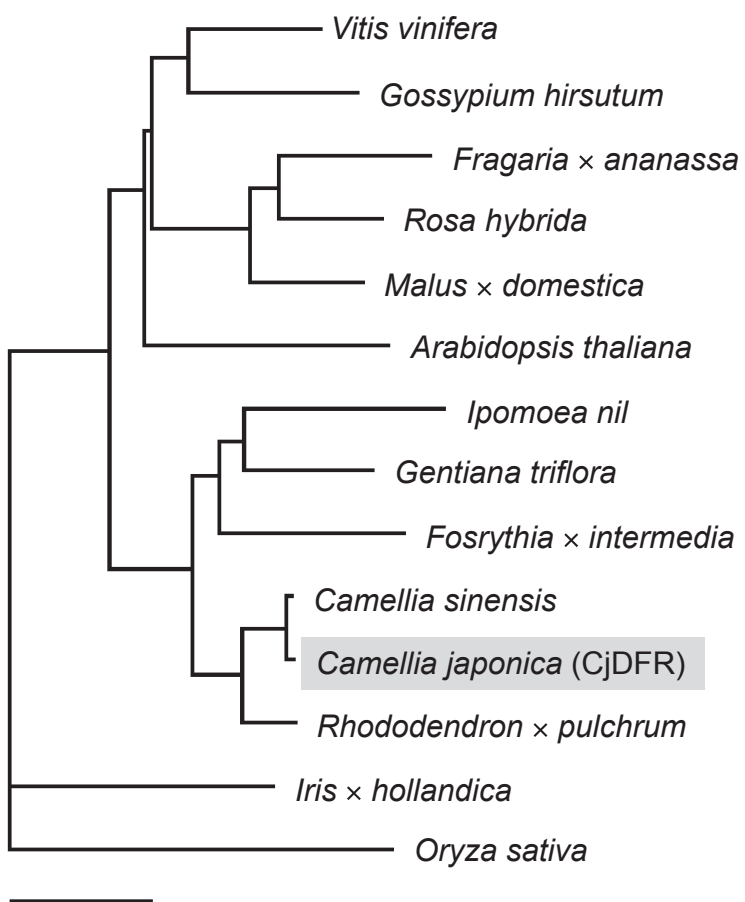

Fig. 6. A phylogenetic tree for DFR constructed by neighbor-joining methods using partial deduced amino acid sequences, including CjDFR (AB524885) identified in this study. Sequence data were collected from GenBank databases [Arabidopsis thaliana (AB033294), Camellia sinensis (AB018685), Fosrythia $\times$ intermedia (Y09127), Fragaria $\times$ ananassa (AY695812) Gentiana triflora (D85185), Gossypium hirsutum (EF187441), Ipomoea nil (AB006792), Iris $\times$ hollandica (AB304917), Malus $\times$ domes tica (AY227728), Oryza sativa (AB003496) Rhododendron $\times$ pulchrum (AB289595), Rosa hybrida (D85102), Vitis vinifera (X75964)].

0.1

\begin{tabular}{|c|c|c|c|c|c|}
\hline 10 & 20 & 30 & 40 & 50 & 60 \\
\hline АССTTGTCTT & СССTGAAGAC & AAGCGTGACA & TGTCCATTTG & GCCTAAGACA & CCATCCGACT \\
\hline 70 & 80 & 90 & 100 & 110 & 120 \\
\hline ATATTCCGGC & AACAAGCGAG & $\begin{array}{r}\text { TACGCAAAGC } \\
C \mathbf{C} A\end{array}$ & $\begin{array}{l}\text { AACTACGAGG } \\
\text { INS F }\end{array}$ & TCTAGCAACA & AAAGTCCTGT \\
\hline 130 & 140 & 150 & 160 & 170 & 180 \\
\hline CGGCССTCTC & ACTCGGCTTG & GGACTAGAAG & AAGGCCGACT & AGAAAAAGAA & GTAGGAGGCA \\
\hline 190 & 200 & 210 & 220 & 230 & 240 \\
\hline TGGAAGAGCT & GСАTCTCCAA & ATGAAAATAA & АСТАТТАССС & AAAATGCCCT & CAGCCAGAGC \\
\hline 250 & 260 & 270 & 280 & 290 & 300 \\
\hline TCGCCCTCGG & CGTCGAAGCC & CACACCGACG & TСTСTGCCCT & САССТТСАТС & СТССАСААСА \\
\hline 310 & 320 & 330 & 340 & 350 & 360 \\
\hline TGGTTCCCGG & ССТGCAАСТС & TTCTACGAGG & GCAAATGGAT & САСТGCCAAA & TGCGTCCCCA \\
\hline 370 & 380 & 390 & 400 & 410 & 420 \\
\hline АСТССАТТАТ & CATGCACATT & GGTGACACGG & TAGAAATTCT & CAGTAACCGC & AAGTACAAGA \\
\hline 430 & 440 & ${ } j A N S \mathrm{R}_{450}$ & 460 & 470 & \\
\hline
\end{tabular}

Fig. 7. Nucleotide sequence of partial cDNA of CjANS. The shadowed boxes represent the primer regions of $C j A N S \mathrm{~F}$ and $\mathrm{R}$ used in this study.

primer pairs (CjF3Ha, 357bp; CjDFR, 412bp; CjANS, 312bp) (Fig. 10).

\section{DISCUSSION}

We identified the partial cDNA sequences of $C j F 3 H$, CjDFR and CjANS. Their deduced amino acid sequences showed high identities with those of woody plants (Figs.
3, 6 and 9) and some amino acid residues important for their catalytic activities were conserved in the sequences obtained in this study (Figs. 2, 5 and 8). These facts support that the genes we identified were the homologues of C. japonica.

Gene-specific primer pairs were constructed to amplify the parts of three anthocyanin biosynthetic genes, CjF3Ha, CjDFR and CjANS (Figs. 1, 4, 7 and Table 1). 


\begin{tabular}{|c|c|c|c|c|c|c|c|}
\hline & & 10 & 20 & 30 & 40 & 50 & \\
\hline CjANS & 1 & LVFPEDKRDM & SIWPKTPSDY & IPATSEYAKQ & LRGLATKVLS & ALSLGLGLEE & 50 \\
\hline Vitis & 1 & LIFPEDKRDM & TIWPKTPSDY & VPATCEYSVK & LRSLATKILS & VLSLGLGLEE & 50 \\
\hline Malus & 1 & CVYPEDKRDL & SIWPQTPADY & IEATAEYAKQ & LRELATKVLK & VLSLGLGLDE & 50 \\
\hline Rosa & 1 & CVYPEDKRDI & SIWPQTPSDY & IVATSEYAKE & LRGLATKILT & VLSLGLGLEE & 50 \\
\hline Gentiana & 1 & CIYPERKRDM & SIWPKTPHDY & IPATIEYAKQ & LRDLATKVLA & VLSVGLGLEP & 50 \\
\hline Ipomoea nil & 1 & CIFPEDKTDL & SIWPKTPSDY & IDATKEYAKQ & LRALATKVLA & VLSLGLGLEE & 50 \\
\hline Arabidopsis & 1 & NYLPSSIRNP & SKWPSQPPKI & RELIEKYGEE & VRKLCERLTE & TLSESLGLKP & 50 \\
\hline \multirow[t]{3}{*}{ Oryza sativa } & 1 & LVHPDHLADH & SLWPANPPEY & VPVSRDFGGR & VRTLASKLLA & ILSLGLGLPE & 50 \\
\hline & & $\star$ & $\star \star \quad \star$ & & $\star \star$ & $\star \star \quad \star \star \star \star$ & \\
\hline & & 60 & 70 & 80 & 90 & 100 & \\
\hline CjANS & 51 & GRLEK----- & EVGGMEE-LH & LQMKINYYPK & CPQPELALGV & EAHTDVSALT & 100 \\
\hline Vitis & 51 & GRLEK----- & EVGGMEE-LL & LQKKINYYPK & CPQPELALGV & EAHTDVSALT & 100 \\
\hline Malus & 51 & GRLEK----- & EVGGLEE-LL & LQMKINYYPK & CPQPELALGV & EAHTDVSALT & 100 \\
\hline Rosa & 51 & GRLEK----- & EVGGLEE-LV & LQMKINYYPK & CPQPELALGV & EAHTDISALT & 100 \\
\hline Gentiana & 51 & DRLEN----- & EVGGMEE-MI & LQKKINYYPK & CPQPELALGV & EAHTDVSALT & 100 \\
\hline Ipomoea nil & 51 & GRLEK----- & EVGGMEE-LL & LQMKINYYPK & CPQPELALGV & EAHTDVSALT & 100 \\
\hline Arabidopsis & 51 & NKLMQALGGG & DKVGAS---- & --LRTNFYPK & CPQPQLTLGL & SSHSDPGGIT & 100 \\
\hline \multirow[t]{3}{*}{ Oryza sativa } & 51 & ETLERRLRGH & ELAGVDDDLL & LQLKINYYPR & CPRPDLAVGV & EAHTDVSALS & 100 \\
\hline & & * & $\star$ & $\star \star \star$ & 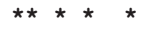 & $\star \star$ & \\
\hline & & 110 & 120 & 130 & 140 & 150 & \\
\hline CjANS & 101 & FILHN-MVPG & LQLFYEGKWI & TAKCVPNSII & MHIGDTVEIL & SNRKYKSILH & 150 \\
\hline Vitis & 101 & FILHN-MVPG & LQLFYEGKWV & TAKCVPNSII & MHIGDTIEIL & SNGKYKSILH & 150 \\
\hline Malus & 101 & FILHN-MVPG & LQLFYEGKWV & TAKCVPNSIV & MHIGDTLEIL & SNGKYKSILH & 150 \\
\hline Rosa & 101 & FILHN-MVPG & LQLFYGGKWV & TAKCVPNSIV & MHIGDTLEIL & SNGKYKSILH & 150 \\
\hline Gentiana & 101 & FILHN-MVPG & LQLFYQGKWI & TAKCVPDSII & MHVGDTLEIL & SNGKYKSILH & 150 \\
\hline Ipomoea nil & 101 & FILHN-MVPG & LQLFYGGKWV & TAKCVPNSII & MHVGDTVEIL & SNGKYKSILH & 150 \\
\hline Arabidopsis & 101 & ILLPDEKVAG & LQVRRGDGWV & TIKSVPNALI & VNIGDQLQIL & SNGIYKSVEH & 150 \\
\hline \multirow[t]{3}{*}{ Oryza sativa } & 101 & FILHN-GVPG & LQVHHAGSWV & TARPEPGTIV & VHVGDÄLEIL & TNGRYTSVLH & 150 \\
\hline & & * $\quad$ * & * $* \quad$ * & * $\quad$ * & $\star \star \star \star * *$ & 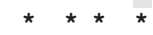 & \\
\hline & & 160 & 170 & 180 & 190 & 200 & \\
\hline CjANS & 151 & RGLVNKEKVR & IS $\ldots \ldots \ldots$ & $\ldots \ldots \ldots$ & $\ldots \ldots \ldots$ & $\ldots \ldots \ldots$ & 200 \\
\hline Vitis & 151 & RGLVNKEKVR & IS $\ldots \ldots$ & $\ldots \ldots \ldots$ & $\ldots \ldots \ldots$ & & 200 \\
\hline Malus & 151 & RGMVNKEKVR & IS.... & $\ldots \ldots \ldots$ & $\ldots \ldots$ & $\ldots \ldots \ldots$ & 200 \\
\hline Rosa & 151 & RGLVNKEKVR & Is $\ldots \ldots \ldots$ & $\ldots \ldots \ldots$ & $\ldots \ldots$ & $\ldots \ldots \ldots$ & 200 \\
\hline Gentiana & 151 & RGLVNKEKVR & Is $\ldots \ldots \ldots$ & $\ldots \ldots \ldots \ldots$ & $\ldots \ldots$ & $\ldots \ldots \ldots$ & 200 \\
\hline Ipomoea nil & 151 & RGVVNREKVR & vs...... & $\ldots \ldots \ldots$ & $\ldots \ldots$ & $\ldots \ldots \ldots$ & 200 \\
\hline Arabidopsis & 151 & QVIVNSGMER & vs ...... & & & & 200 \\
\hline Oryza sativa & 151 & RGLVSRDAVR & Ls $\ldots \ldots \ldots$ & $\ldots \ldots \ldots$ & $\ldots \ldots$ & $\ldots \ldots \ldots$ & 200 \\
\hline
\end{tabular}

Fig. 8. Comparison of the deduced amino acid sequence of partial CjANS and those of corresponding parts of other plant species. Asterisks indicate completely identical amino acid residues among eight species. The shadowed amino acid residues (His93, Asp95 and His150) represent absolute conservation important for catalytic activity. Species names and their GenBank accession numbers are as follows; Arabidopsis (Arabidopsis thaliana, AY093302); Gentiana (Gentiana triflora, AB193310); Ipomoea nil (Ipomoea nil, AB073920); Malus (Malus $\times$ domestica, AB074487); Oryza sativa (Oryza sativa, Y07955); Rosa (Rosa hybrida, AB239787); Vitis (Vitis vinifera, EF192468).

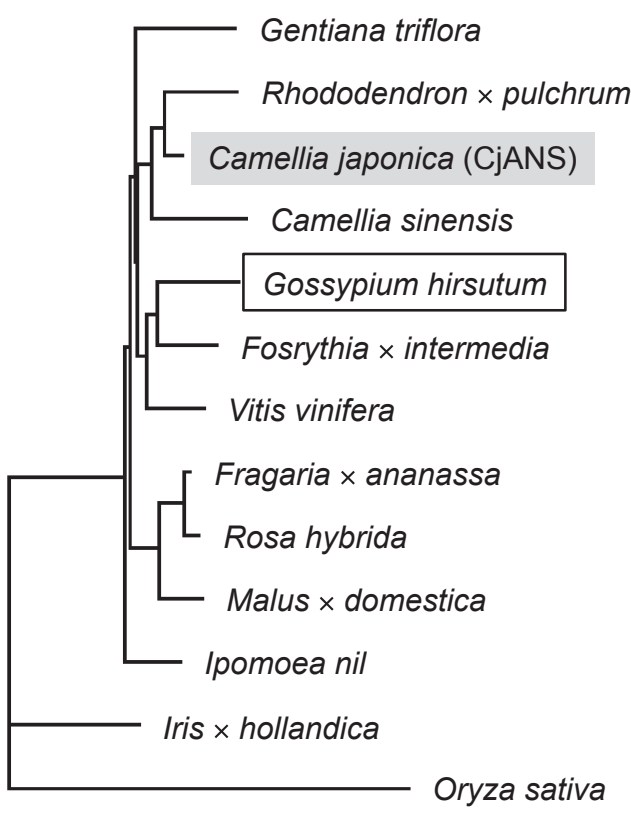

Fig. 9. A phylogenetic tree for ANS constructed by neighbor-joining methods using partial deduced amino acid sequences, including CjANS (AB524886) identified in this study. Sequence data were collected from GenBank databases [Camellia sinensis (AY830416), Fosrythia $\times$ intermedia (Y12489), Fragaria $\times$ ananassa (AY695817), Gentiana triflora (AB193310), Gossypium hirsutum (EF187442), Ipomoea nil (AB073920), Iris $\times$ hollandica (AB284174), Malus $\times$ domestica (AB074487), Oryza sativa (Y07955), Rhododendron $\times$ pulchrum (AB289596), Rosa hybrida (AB239787), Vitis vinifera (EF192468)]. 


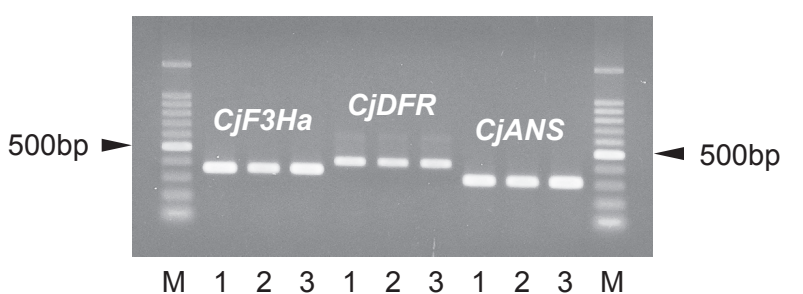

Fig. 10. RT-PCR products of CjF3Ha, CjDFR and CjANS using gene-specific primer pairs designed in this study. M indicates 100 bp DNA Ladder Marker. 1, wild type C. japonica; 2, 'Tamanoura'; 3, 'Hatsu-arashi'. Three independent flowers were analysed per each accession.

They successfully amplified single PCR products of expected sizes (Fig. 10). It was, therefore, shown that primer pairs designed here are applicable to investigate the expression profiles of these genes on petal pigmentation of C. japonica.

Insertion or excision of transposable elements on anthocyanin biosynthetic genes caused flower colour mutation, like fleck and sector in the genus Ipomoea (Iida et al., 2004). Camellia japonica has been domesticated for a long time in Japan (Tuyama, 1968a), and there are many cultivars bearing flowers which imply such involvements of genetic mutations (Tuyama, 1968b). Molecular information we presented here is useful to elucidate the mechanism of such flower colour mutation in C. japonica.

Camellia japonica 'Hatsu-arashi' lacks an accumulation of anthocyanins and results in generating a white coloured flower (Savige, 1993). However, the expression levels of three genes tested in its petals showed no differences from those of wild type C. japonica and 'Tamanoura' (red parts of the petals) (Fig. 10). One possibility is that other anthocyanin biosynthetic genes except $C j F 3 H, C j D F R$ and CjANS might be down-regulated. An alternative is an involvement of mutations caused by nucleotide substitution or insertion/deletion, if some of the above three genes are responsible. These mutations induce an amino acid substitution or frame shift translation, which are responsible for loss of enzymatic activities, although mRNA transcriptional levels are not suppressed, as in the case of Matthiola incana (Hemleben et al., 2004).

\section{ACKNOWLEDGEMENTS}

This study was supported by Research Fellowships of the Japan Society for the Promotion of Science for Young Scientists. We also acknowledge a financial support from The Otomo Fund of The International Camellia Society (ICS).

\section{REFERENCES}

Davies, K. M. 2009 Modifying anthocyanin production in flowers. In "Anthocyanins", ed. by K. Gould, K. Davies and C. Winefield, Springer, New York, pp. 49-83

Deroles, S. 2009 Anthocyanin biosynthesis in plant cell cultures: a potential source of natural colourants. In "Anthocyanins", ed. by K. Gould, K. Davies and C. Winefield, Springer, New York, pp. 107-167

Felsenstein, J. 2004 PHYLIP (Phylogeny Inference Package) version 3.6, Department of Genome Sciences, University of Washington, Seattle, U.S.

Glover, B. J. 2007 Understanding Flowers and Flowering. Oxford Univ. Press Inc., New York.

Hemleben, V., A. Dressel, B. Epping, R. Lukacin, S. Martens and M. B. Austin 2004 Characterization and structural features of a chalcone synthase mutation in a white-flowering line of Matthiola incana R. Br. (Brassicaceae). Plant Mol. Biol., 55: 455-465

Holton, T. A. and E. C. Cornish 1995 Genetics and biochemistry of anthocyanin biosynthesis. Plant Cell, 7: 1071-1083

Iida, S., Y. Morita, J. D. Choi, K. I. Park and A. Hoshino 2004 Genetics and epigenetics in flower pigmentation associated with transposable elements in morning glories. Adv. Biophys., 38: $141-159$

Kiefer, E., W. Heller and D. Ernst 2000 A simple and efficient protocol for isolation of functional RNA from plant tissues rich in secondary metabolites. Plant Mol. Biol. Rep., 18: 33-39

Martin, C., A. Prescott, S. Mackay, J. Bartlett and E. Vrijlandt 1991 Control of anthocyanin biosynthesis in flowers of Antirrhinum majus. Plant J., 1: 37-49

Sakata, Y., K. Arisumi and I. Miyajima 1986 Cyanidin 3-galactoside, a new anthocyanin from Camellia japonica subsp. rusticana (Honda) Kitamura and its occurrence in the garden forms of Camellia of Japanese origin. J. Japan. Soc. Hort. Sci., 55: $82-88$

Sakata, Y., K. Arisumi and I. Miyajima 1987 Constitution of anthocyanins in flowers of the wild forms of section camellia of Japanese and Formosan origin. J. Japan. Soc. Hort. Sci., 56: 208-214

Savige, T. J. 1993 The International CAMELLIA REGISTER. Fine Arts Press Pty. Ltd., Sydney, p. 781

Tuyama, T. 1968a Camellias of Japan. Hirokawa Publishing Co. Ltd., Tokyo, pp. 1-2

Tuyama, T. 1968b Camellias of Japan Color Plates. Hirokawa Publishing Co. Ltd., Tokyo, pp. 160-200 